\title{
Numerical simulation of natural convection in square cavities with power function temperature wall by lattice Boltzmann method
}

\author{
Xin $\operatorname{Tian}^{1, a}$, Zhengwei Shui ${ }^{2, b}$ and Chiyuan $\operatorname{Ren}^{3, c}$ \\ ${ }^{1}$ Apply Technique College, Southwest Petroleum University, Chengdu, 610500, China \\ ${ }^{2}$ Apply Technique College, Southwest Petroleum University, Chengdu, 610500, China \\ ${ }^{3}$ Science College, Southwest Petroleum University, Chengdu, 610500, China \\ aemail: yuxin0824@126.com, bemail: 47382884@qq.com, email: renchiyuan@163.com
}

Keywords: Natural convection; Square cavity; Power function temperature boundary; Lattice Boltzmann model

\begin{abstract}
In this study, a thermal lattice Boltzmann model is employed to investigate the natural convection in square cavities with power function temperature boundary. The horizontal wall of cavities is adiabatic, and the temperature at the west and east wall is set to constant or a power function. Four cases classified by temperature condition are investigated in detail at different Rayleigh number, 103,104,105,106 and power index, 0, 0.5, 1, 2, 4 by Nusselt number and some hydrodynamic variables. Results show: though the average temperature different between west and east wall is same in the four cases on given power index, the thermal and hydrodynamic performance are of notable different, by which a useful suggestion about heat transfer rate in square cavities is put forward.
\end{abstract}

\section{Introduction}

Natural convection in closed enclosures has induced many researchers' interest due to significant industrial applications such as melting process, material processing, cooling systems for electronic devices etc. [1-4]. Natural convection in industry can be used in different conditions, such as different boundary condition, enclosure shape, or filled media, so a number of numerical and experimental studies have been carried for natural convection under different condition.

The studies of natural convection in different shape enclosures have been investigated widely, including rectangular, cubic triangular, cylinder and so on. Natural convection in rectangular is the most normal case, the research in which is thorough. A large cross section of fundamental research on this topic has been reviewed by Catton [5] and Bejan [6]. Davis [7] made a bench solution in square cavity with Rayleigh number up to 106.HortmannandPeric[8] further the accuracy by finite volume and multi-grid technology for square cavity with heat left wall, cool right wall, and insulated top and bottom walls. Dillon, Emery, Mescher [9] made a deeply investigation for tall cavity with aspect ratios $8,15,20$, and 33 .Besides rectangular cavity, the triangular enclosure is usually studies under different boundary condition in recently years. Saha and Khan [10] studied natural convection within a tilted isosceles triangular enclosure with discrete bottom heating. And Many researchers paid their attention on inclined enclosures. Basak et al. [11] have studied the natural convection in isosceles triangular enclosures via heatline analysis for linear heating of inclined walls. Heo, Chung [12] made an experimental investigation of natural convection heat transferon inclined cylinders for a range of Rayleigh numbers and inclined angle.

In recently years, natural convection in cavities filled with different mediahas been investigated, which include porous media, magnetic media, nanofluid. Vadasz[13] took an investigation to heat flux dispersion in natural convection in porous media.Amaguchi et al. [14]investigated the natural convection filled with magnetic fluid in a rectangular box.Oztop et al. [15] numerically analysis steady state natural convection in an enclosure filled with nanofluid.

A number of investigations to natural convection with different temperature boundary condition have been performed. Sathiyamoorthya et al. [16] made a numerical study to natural convection 
flow in a closed square cavity when the bottom wall is uniformly heated and vertical wall(s) are linearly heated. V. Sivakumar et al. [17] analyzed the mixed convection in cavities with different lengths of the heating portion and different locations. Mahapatra et al. [18] investigated the effects of sine heating of wall on double-diffusive natural convection in square cavities.

\section{Mathematic formulation and solution procedure}

\section{Problem statement}

The geometry of the present problem is shown in Fig1. It displays a two-dimension enclosure with the height $\mathrm{H}$. The temperatures of the west and east wall are set to $T h, T c_{\text {or a power function }}$ of height ${ }^{y}$. In this article, $T h$ and $T c$ are set to 1 and 0 , respectively. The north and south walls are adiabatic. The enclosure is filled with fluid, which is ideal Newtonian, incompressible and laminar flow. Contrasted with normal natural convection, the main difference is the temperature of vertical walls is not a constant but a power function.

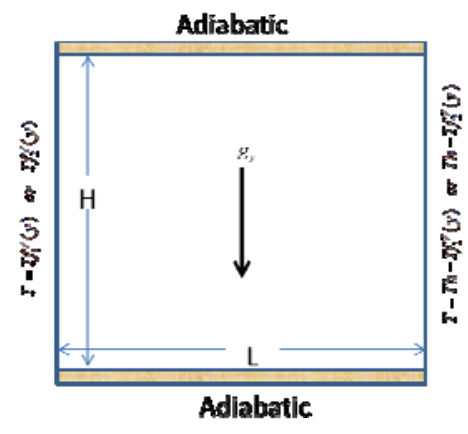

Fig1. Schematic diagram of the physical configuration and the coordinate system

In Fig. $1, g_{y}$ is gravity acceleration, and $T f_{1}^{\gamma}, T f_{2}^{\gamma}$ are power functions with index $\gamma$, defined as

$$
T f_{1}^{\gamma}(y)=T_{h} \cdot\left(\frac{y}{H}\right)^{\gamma} \quad \text { (1) } \quad T f_{2}^{\gamma}(y)=T_{h} \cdot\left(1-\frac{y}{H}\right)^{\gamma}
$$

where $\gamma$ is power index. It is easy to be computed that let $\gamma \rightarrow 0$, then $T f_{1}^{\gamma}, T f_{2}^{\gamma} \rightarrow T h$, and let $\gamma \rightarrow \infty$, then $T f_{1}^{\gamma}, T f_{2}^{\gamma} \rightarrow 0$. Specially, let $\gamma=1$, then $T f_{1}^{\gamma}, T f_{2}^{\gamma}$ are linear function.

\section{Lattice Boltzmann method}

For the incompressible, the thermal lattice Boltzmann equation adopts a uniform lattice with BGK collision model. The two distribution functions model may be expressed as:

For the flow field:

$$
f_{i}\left(x+e_{i} \Delta t, t+\Delta t\right)=f_{i}(x, t)-\frac{1}{\tau_{f}}\left[f_{i}(x, t)-f_{i}^{e q}(x, t)\right]+\Delta t F_{i}
$$

For the temperature field:

$$
g_{i}\left(x+e_{i} \Delta t, t+\Delta t\right)=g_{i}(x, t)-\frac{1}{\tau_{g}}\left[g_{i}(x, t)-g_{i}^{e q}(x, t)\right]
$$

where $f_{i}$ and $g_{i}$ are the particle density and energy distribution function along the particle velocity direction $e_{i}$, respectively, $f_{i}^{e q}$ and $g_{i}^{e q}$ are equilibrium distribution function, and $\tau_{f}$ and $\tau_{g}$ are the dimensionless relaxation times that control the rates approaching equilibrium. Also, $F_{i}$ is an external force term along velocity direction $e_{i}$. 


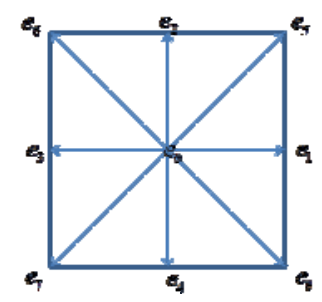

Fig2. Discrete velocity vectors for D2Q9 model

The equilibrium distribution functions, which depend on the local density and velocity, are given by the form

$$
\begin{aligned}
& f_{i}^{e q}=w_{i} \rho\left[1+\frac{e_{i} \cdot u}{c^{2}}+\frac{\left(e_{i} \cdot u\right)^{2}}{2 c^{4}}-\frac{u \cdot u}{2 c^{2}}\right] \\
& g_{i}^{e q}=w_{i} \rho T\left[1+\frac{e_{i} \cdot u}{c^{2}}+\frac{\left(e_{i} \cdot u\right)^{2}}{2 c^{4}}-\frac{u \cdot u}{2 c^{2}}\right]
\end{aligned}
$$

where $u$ and $\rho$ are the macroscopic velocity and density, respectively, $c=\frac{\Delta x}{\Delta t}$ the lattice speed where $\Delta x$ and $\Delta t$ are set as unit, $w_{i}$ is the weighting factor.

For D2Q9 model, the speed vector $e_{i}$ and weight $w_{i}$ are set as follows:

$$
\begin{aligned}
& e_{i}= \begin{cases}0 & i=0 \\
\left.c\left(\cos \left((i-1) \frac{\pi}{2}\right), \sin \left((i-1) \frac{\pi}{2}\right)\right)\right) & i=1-4 \\
\left.\frac{\sqrt{2}}{2} c\left(\cos \left(\left(i-\frac{9}{2}\right) \frac{\pi}{2}\right), \sin \left(\left(i-\frac{9}{2}\right) \frac{\pi}{2}\right)\right)\right) & i=5-8\end{cases} \\
& w_{i}= \begin{cases}\frac{4}{9} & i=0 \\
\frac{1}{9} & i=1-4 \\
1 / 36 & i=5-8\end{cases}
\end{aligned}
$$

The corresponding kinematic viscosity and thermal diffusivity are then related to the relation times by:

$$
\begin{aligned}
& v=\left[\tau_{f}-\frac{1}{2}\right] c_{s}^{2} \Delta t \\
& \alpha=\left[\tau_{g}-\frac{1}{2}\right] c_{s}^{2} \Delta t .
\end{aligned}
$$

where $c_{s}$ is lattice sound speed in media, it is equals to $c_{s}=\frac{c}{\sqrt{3}}$.

As usual, in order to incorporate buoyancy force in the model, the Boussinesq approximation was applied, therefore the force term in the Eq [12] need to be calculated as below in vertical $\operatorname{direction}(\mathrm{y})$ :

$$
F_{y}=3 w_{k} g_{y} \beta\left(T-T_{m}\right)
$$

Where $g_{y}$ is the acceleration of gravity acting in the $y$ direction of the lattice links; $\beta$ is the thermal expansion coefficient and $T_{m}$ is reference temperature. 


\section{Result and discussion}

We use a uniform mesh of size $N x \times N y=N^{2}$ with $N^{2}$ equal to $32^{2}, 64^{2}, 128^{2}, 256^{2}$ to investigate the grid independence by Nusselt number $N u_{\text {avg }}$ and $N u_{0}$. In table [1] we tabulate the Nusselt number on different grids with power index $\gamma=0$ in case I. From the table, we can clearly see that when $N$ increases, the calculated average Nusselt quickly approaches the benchmark result. When $N$ further increases from 128 to 256, there is not much improvement for the result. To short computing time, we set grids as $128^{2}$ in following simulation.

To verify our codes validity, our result is compared with the benchmarks given by Hortmann[8], Davis[19], Mayne[20]. Table [2] shows the average Nusselt number $N u_{\text {avg }}$, the maximum horizontal velocity on the vertical midplane of the cavity $u_{\text {max }}^{x}$, and the maximum vertical velocity on the horizontal midplane of the cavity $u_{\text {max }}^{y}$.It can be seen from table [1], that our result agrees well with the benchmarks.

Table 1 Grid dependence study

\begin{tabular}{|l|l|l|l|l|}
\hline Ra & $\mathbf{1 0}^{\mathbf{3}}$ & $\mathbf{1 0}^{\mathbf{4}}$ & $\mathbf{1 0}^{\mathbf{5}}$ & $\mathbf{1 0}^{\mathbf{6}}$ \\
\hline grids & $N u_{\text {avg }}$ & & & \\
\hline $\mathbf{3 2}^{2}$ & 1.1363 & 2.2636 & 4.5320 & 8.8445 \\
\hline $\mathbf{6 4}^{2}$ & 1.1308 & 2.2624 & 4.5307 & 8.8389 \\
\hline $\mathbf{1 2 8}^{\mathbf{2}}$ & 1.1225 & 2.2541 & 4.5219 & 8.8031 \\
\hline $\mathbf{2 5 6}^{\mathbf{2}}$ & 1.1208 & 2.2446 & 4.5187 & 8.8190 \\
\hline grids & $u_{\text {max }}^{x}$ & & & \\
\hline $\mathbf{3 2}^{\mathbf{2}}$ & 3.6244 & 15.9956 & 34.7401 & 64.6905 \\
\hline $\mathbf{6 4}^{2}$ & 3.6109 & 16.1123 & 34.7459 & 64.6771 \\
\hline $\mathbf{1 2 8}^{2}$ & 3.6051 & 16.1561 & 34.7209 & 64.6537 \\
\hline $\mathbf{2 5 6}^{2}$ & 3.5939 & 16.2274 & 34.7116 & 64.6468 \\
\hline grids $^{\mathbf{2}}$ & $u_{\text {max }}^{y}$ & & & \\
\hline $\mathbf{3 2}^{\mathbf{2}}$ & 3.7048 & 19.4691 & 68.9051 & 219.6021 \\
\hline $\mathbf{6 4}^{\mathbf{2}}$ & 3.6915 & 19.5567 & 68.7446 & 219.5949 \\
\hline $\mathbf{1 2 8}^{\mathbf{2}}$ & 3.6891 & 19.6296 & 68.6114 & 219.5016 \\
\hline $\mathbf{2 5 6}^{\mathbf{2}}$ & 3.6830 & 19.6540 & 68.6225 & 219.4843 \\
\hline
\end{tabular}

Table 2 Comparison of Nusselt number and hydrodynamic variables with benchmark solutions.

\begin{tabular}{|l|l|l|l|l|}
\hline $\mathbf{R a}$ & $\mathbf{1 0}^{\mathbf{3}}$ & $\mathbf{1 0}^{\mathbf{4}}$ & $\mathbf{1 0}^{\mathbf{5}}$ & $\mathbf{1 0}^{\mathbf{6}}$ \\
\hline Present & $\mathrm{Nu}$ avg & & & \\
\hline ref [8] & 1.1225 & 2.2541 & 4.5219 & 8.8031 \\
\hline ref [19] & - & 2.243 & 4.519 & 8.800 \\
\hline ref[20] & - & - & - & - \\
\hline & $u_{\max }^{x}$ & & & \\
\hline Present & 3.6051 & 16.1561 & 34.7209 & 64.6537 \\
\hline ref [9] & 3.649 & 16.178 & 34.73 & 64.63 \\
\hline ref [19] & - & 16.180 & 34.740 & 64.834 \\
\hline ref[20] & 3.649 & - & - & - \\
\hline & $u_{\max }^{y}$ & & & \\
\hline Present & 3.6891 & 19.6296 & 68.6114 & 219.5016 \\
\hline ref [8] & 3.697 & 19.617 & 68.59 & 219.36 \\
\hline ref [19] & - & 19.630 & 68.640 & 220.461 \\
\hline ref[20] & 3.696 & - & - & - \\
\hline
\end{tabular}




\section{Conclusion}

In this work we employ the lattice Boltzmann method to investigate natural convection with power function temperature wall in square cavities. The coupled double distribution model is employed to simulation the flow and heat transfer: a D2Q9 scheme for mass and momentum conservation equation, and another D2Q9 scheme for the advection-diffusion equation for the temperature. This approach is valid under the Boussinesq approximation. The natural convection with power function type temperature wall is classified to four cases by different temperature setting on the vertical wall. Detail investigation is performed under different parameters value in cases.

We systematically study the validity and grid impendence of codes. The results show our codes are valid to realize the simulation by comparison with benchmark solutions.

By this investigation, a useful conclusion for natural convection in cavity can be make: if the average temperature difference between vertical walls is set as constant, the higher the temperature difference in the top is, the less the heat transfer effective is.

\section{References}

[1] P.O. Iwanik, W.K.S. Chiu, Temperature distribution of an optical fiber traversing through a chemical vapor deposition reactor, Num. Heat Transf. Part A Appl. 43(2003) 221-237.

[2] L.A. Florio, A. Harnoy, Combination technique for improving natural convection cooling in electronics, Int. J. Therm. Sci. 46(2007) 76-92.

[3] L.K. Saha, M.A. Hossain, R.S.R. Corla, Effect of Hall current on the MHD laminar natural convection flow from a vertical permeable flat plate with uniform surface temperature, Int. J. Therm. Sci. 46(2007) 790-801.

[4] Y.Y Yan, H.B. Zhang, J.B. Hull, Numerical modeling of electrohydrodynamic (EHD) effect on natural convection in an enclosure, Num. Heat Transf. Part A Appl. 46(2004) 453-471.

[5] I. Catton, Natural convection in enclosures. Proc. 6th Int. Heat Transfer Conf. 6(1978) 13-31.

[6] A. Bajan, convection Heat Transfer. John Wiley, New York, 2004.

[7] G. de Vahl Davis, Natural convection of air in a square cavity: a bench mark numerical solution, Int. Numer. Methods Fluids 3(1983) 249-264.

[8]M.Hortmann, M. Peric, G. Scheuerer, Finite volume multigrid prediction of laminar natural convection: bench-mark solutions,Int. J.Numer. Meth.Fluids. 11(1990) 189-207.

[9] H.E. Dillon, A. Emery, A. Mescher, Benchmark Comparison of Natural Convection in a Tall Cavity, COMSOL Conference. 2009. Boston.

[10] S.C. Saha, M.M.K. Khan, A review of natural convection and heat transfer in attic-shaped space, Energy and Buildings 43(2011) 357-365.

[11] T. Basak, R. Anandalakshmi, Monisha Roy, Heatlines based natural convection analysisi in tilted isosceles triangular enclosures with linearly heated inclinded walls: effect of various orientations. Int. Comm. Heat \& Mass Transf, 43(2013) 39-45.

[12] J.H. Heo, B.J. Chung, Natural convection heat transfer on the outer surface of inclined cylinders, Natural convection heat transfer on the outer surface of inclined cylinders, 73 (2012) $366-372$.

[13]P. Vadasz, Heat flux dispersion in natural convection in porous media, Int. J. Heat \& Mass Transfer, 53(2010), 3394-3404.

[14] H Yamaguchi, I Kobori, Y Uehata, K Shimada, Natural convection of magnetic fluid in a rectangular box, J. Magnetism and Magnetic Materials, 201(1999), 264-267. 
[15] H.F. Oztop, E.A. nada, Y. Varol, K.A. Salem, Computational analysis of non-isothermal temperature distribution on natural convection in nanofluid filled enclosures, Superlattices and Microstructures, 49(2011), 453-467.

[16] M. Sathiyamoorthya, T. Basakb, S.Roy, Y. Pop, Steady natural convection flows in a square cavity with linearly heated side wall(s), Int. J. Heat \& Mass Transfer, 50(2007),766-775.

[17] V. Sivakumar, S. Sivasankaran, P. Prakash, Jinho Lee, Effect of heating location and size on mixed convection in lid-driven cavities. 59(2010) 3053-3065.

[18]Tapas Ray Mahapatra, Dolal Pal, SabyasachiMondal, Effects of buoyancy ratio on double-diffusive natural convection in a lid-driven cavity, Int. J. Heat \& Mass Transf. 57(2013) 771-785.

[19] G. de Vahl Davis, I.P. Jones, Natural convection in a square cavity: a comparison exercise, Int. J. Numer. Meth.Fluid 3(1983) 227-248.

[20] D.Mayne, A.S. Usmani,M. Crapper, h-adaptive finite element soliution of high Rayleigh number thermally driven cavity problem, Int. J. Numer.Meth.Heat Fluid Flow. 10(2000), 598-615. 